REVIEW ARTICLE

\title{
Obesity in Pregnancy: A New Chapter in Obstetrics
}

\author{
Aris Antsaklis
}

\begin{abstract}
Obesity is a worldwide health problem affecting more than $35 \%$ of the adult population in the USA. Obesity is the greatest epidemic ever experienced by humans and resulting from increasing population increasing lifespan, urbanization, plentiful food and physical inactivity. The rate of obesity has doubled over the past decade. Percentage of women who are overweight or obese has increased by $60 \%$ over the past 30 years. The mean BMI has increased over the past 20 years leading to adverse metabolic effects on blood pressure, cholesterol and triglyceride concentration and insulin resistance, thereby increasing the risk: (1) for coronary disease and ischemic stroke, (2) for type 2 diabetes, and (3) for polycystic ovary syndrome (PCOS). Maternal obesity in pregnancy (MOP) has been associated with fertility implications both genders pregnancy complication, such as preterm delivery, shoulder dystocia, and adverse outcome including hypertensive disorders, gestational diabetes and need for operative delivery (cesarean section and instrumental vaginal delivery). Maternal obesity also has a significant impact on fetal development on neonatal period and overall on childhood development. Modification to routine prenatal care has been suggested for this population such as screening for diabetes in early pregnancy, limiting gestational weight gain, routine ultrasound for gestational age, fetal anatomic survey and antenatal surveillance with NST and BPP scoring and fetal echocardiography, screening for fetal aneuploidy. Obesity can affect screening test performance. Cell-free fetal DNA screening is more likely to result in test failure, low-dose aspirin to reduce the risk of preeclampsia and evaluation by anesthesiologist. Delivery timing and indications for labor induction should not be altered based on maternal obesity. For women undergoing cesarean delivery, prophylactic antibiotics should be administered based on maternal weight.
\end{abstract}

Keywords: Cesarean section, Diabetes, Macrosomia, Obesity epidemic.

Donald School Journal of Ultrasound in Obstetrics and Gynecology (2021): 10.5005/jp-journals-10009-1679

\section{INTRODUCTION}

Obesity is a worldwide health problem affecting more than $35 \%$ of the adult population in the USA. Obesity is the greatest epidemic ever experienced by humans and resulting from increasing population increasing lifespan, urbanization, plentiful food, and physical inactivity. The rate of obesity has doubled over the past decade. Percentage of women who are overweight or obese has increased by $60 \%$ over the past 30 years. ${ }^{1}$

Obesity is a chronic disease that increasing in prevalence in adults, adolescents and children is defined as a body mass index (BMI) more than $30 \mathrm{~kg} / \mathrm{m}^{2}$ whereas overweight is defined as a BMI between $25.0 \mathrm{~kg} / \mathrm{m}^{2}$ and $29.9 \mathrm{~kg} / \mathrm{m}^{2}$.

Maternal obesity, based on a BMI more than $29.9 \mathrm{~kg} / \mathrm{m}^{2}$, has emerged as an important risk factor in modern obstetrics worldwide. Measuring BMI is the first step to determine the degree of overweight. The BMI is easy to measure, reliable, and correlated with percentage of body fat and body fat mass. ${ }^{1}$

Body mass index provides a better estimate of total body fat compared with body weigh alone. ${ }^{2}$ BMI classifications are based upon risk of cardiovascular disease. ${ }^{3}$ The recommended classifications for BMI adopted by the $\mathrm{NIH}$ and WHO for Caucasian, Hispanic, and Black individuals are:

- Underweight: Less than $18.5 \mathrm{~kg} / \mathrm{m}^{2}$

- Normal weight: More than $18.5-24.9 \mathrm{~kg} / \mathrm{m}^{2}$

- Overweight: More than $25.0-29.9 \mathrm{~kg} / \mathrm{m}^{2}$

- Obesity: More than $30 \mathrm{~kg} / \mathrm{m}^{2}$.

Obesity can further be subclassified into classes:

- Class I: $30.0-34.9 \mathrm{~kg} / \mathrm{m}^{2}$

- Class II: $35.0-39.9 \mathrm{~kg} / \mathrm{m}^{2}$

- Class III: More than $40 \mathrm{~kg} / \mathrm{m}^{2}$ also referred to as severe extreme or massive obesity.
Alexandra Maternity Hospital, University of Athens, Greece

Corresponding Author: Aris Antsaklis, Alexandra Maternity Hospital, University of Athens, Greece, Phone: +306944299699, e-mail: arisants@ otenet.gr

How to cite this article: Antsaklis A. Obesity in Pregnancy: A New Chapter in Obstetrics. Donald School J Ultrasound Obstet Gynecol 2021;15(1):43-48.

Source of support: Nil

Conflict of interest: None

Recently, these categories have been expanded to include an additional category of super obesity (BMI of $>50 \mathrm{~kg} / \mathrm{m}^{2}$ ).

About $35 \%$ of adult women worldwide are estimated to be overweight (BMI > 25) a third of whom (297 million) are obese (BMI $>30 \mathrm{~kg} / \mathrm{m}^{2}$ ). WHO estimates obese and overweight women BMI more than 25 to be $77 \%$ in USA, 73\% in Mexico, 37\% in France, $32 \%$ in China, $69 \%$ in South Africa, and $18 \%$ in India.

In European Region and especially the Eastern Mediterranean Region and the Region of the Americas this proportion exceeds $50 \%$. The mean BMI has increased over the past 20 years leading to adverse metabolic effects on blood pressure, cholesterol and triglyceride concentration and insulin resistance, thereby increasing the risk: (1) for coronary heart disease and ischemic stroke, (2) for type 2 diabetes, and (3) for polycystic ovary syndrome (PCOS).

Adipose tissue is an active endocrine organ and when present in excess, it can have dysregulatory effects on metabolic, vascular, and inflammatory pathways in many organ systems and thereby lead to a variety of reproductive and medical problems. Globally, $44 \%$ of diabetes, $23 \%$ of ischemic heart disease, and $7-41 \%$ for certain cancers, particular breast cancer, attributable to overweight and obesity. 
For example, obesity-related insulin resistance and abnormalities in inflammatory pathways can affect placental growth and function, and have been linked to development of preeclampsia. ${ }^{4}$ Epigenetic changes induced by fetal exposure to increased levels of glucose, insulin, lipids, and inflammatory cytokines may play a role in the long-term outcome of offspring. These in utero effects may result in permanent or transient changes in metabolic programming, leading to adverse health outcomes in adult life (fetal origins of adult disease theory, Barker hypothesis). ${ }^{5,6}$

The obesity epidemic extends to the pregnant population, with $40 \%$ of women qualifying as either overweight or obese, and $28 \%$ of pregnant women qualifying as obese. Causes for maternal obesity are complex and multifactorial. Societal factors such as lack of knowledge regarding nutrition, lack of access to healthy food and limited opportunities for physical activity have negative impacts on maternal and fetal wellbeing.

Maternal obesity in pregnancy (MOP) has been associated with fertility implications both genders pregnancy complication, such as preterm delivery, shoulder dystocia, and adverse outcome including hypertensive disorders, gestational diabetes and need for operative delivery (cesarean section and instrumental vaginal delivery). ${ }^{7}$

Maternal obesity also has a significant impact on fetal development on neonatal period and overall on childhood development. 8

More women enter pregnancy with a BMI more than $30 \mathrm{~kg} / \mathrm{m}^{2}$ leading to an increased risk of complications during pregnancy and delivery. Their infants tend to be born larger and are at greater risk of becoming obese and developing type 2 diabetes as children and adolescents. These women also tend to retain more weight after birth.

There is increasing evidence that obesity has its origin in early life. Predisposition is based on interactions between the genome and environmental influences acting through epigenetic modifications. Individuals most at risk are those whose ancestral line has made a rapid transition from traditional to a westernized style of life. This process involves not only metabolism but also behavior. In recent years, there has been growing interest on how in utero exposures predispose infants to diseases throughout the life span.

David Barker reported that people with a history of low-birth weight were at elevated risk of coronary artery disease later in life and theorized that the origins of complex diseases may stem from intrauterine exposures (Barker hypothesis). Thus, MOP may significantly affect life ex utero for years to come. ${ }^{9-11}$

Obesity is associated with increased risk of almost all pregnant complications, such as gestational hypertension, preeclampsia, gestational diabetes mellitus (GDM), delivery of a large for gestational age (LGA) infant, and a higher incidence of congenital defects all occur more frequently than in women with normal BMI. ${ }^{12}$ Cesarean section rates are higher and anesthesia may be problematic and spontaneous preterm labor.

\section{Maternal Complications of Obesity in PREgnanCy}

\section{Antepartum Complications of Obesity in Pregnancy}

Early Pregnancy Loss and Stillbirth

In a 2011 systematic review including six retrospective studies and a total of 28,538 women [3,800 obese (BMI $>28$ or $\left.30 \mathrm{~kg} / \mathrm{m}^{2}\right), 3,792$ overweight (BMI $\left.25-29 \mathrm{~kg} / \mathrm{m}^{2}\right)$, and 17,146 normal weight $(\mathrm{BMI}<25$ $\left.\left.\mathrm{kg} / \mathrm{m}^{2}\right)\right]$, the percentages of spontaneously conceiving women with more than one miscarriage were $16.6 \%$ for obese women, $11.8 \%$ for overweight women, and $10.7 \%$ for normal-weight women. ${ }^{13,14}$

The cohort of women with recurrent early miscarriage was small but showed a higher risk for recurrent early miscarriage in obese vs normal-BMI women [0.4 vs $0.1 \%$, odds ratio (OR) 3.51 , 95\% confidence interval (CI) 1.03-12.01]. Differences in patient characteristics and study designs, however, limit the validity of these findings. If the early excess loss of euploid embryos among overweight and obese women is confirmed, one mechanism may be an unfavorable hormonal environment related to obesity. Another mechanism may involve inflammatory changes related to PCOS. PCO has been associated with miscarriage rate $20-40 \%$ higher than the baseline in the general obstetric population. ${ }^{15,16}$

A stronger link has been demonstrated between obesity and stillbirth with one meta-analysis showing more than twice the risk of stillbirth compared with patients with normal BMI. ${ }^{17}$

Take-home message is that increased risk of miscarriage in obese women is due to: (1) Impaired folliculogenesis and poor oocyte quality, (2) Impaired endometrial receptivity, (3) A higher prevalence of PCOS among overweight and obese women, and (4) ART can help to select healthy embryo.

The British Fertility Society guidance suggests that fertility treatment should be deferred until BMI is less than $35 \mathrm{~kg} / \mathrm{m}^{2}$.

\section{Pregnancy-associated Hypertension}

There is an association between obesity and hypertensive disorders during pregnancy. Several observational studies demonstrate an association between obesity and gestational hypertension with a reported 2.5-fold to 3.2-fold increased risk. ${ }^{18}$

Maternal weight and BMI are independent risk factor for preeclampsia, as well other hypertensive disorders. ${ }^{19,20}$

Obesity contributes to hypertension by multiple mechanisms: (1) By reduction of available nitric oxide due to oxidative stress, due to increased inflammation and free fatty acids, and lower concentration of circulating antioxidants; (2) By increase of sympathetic tone; and (3) By increased release of angiotensin gen by adipose tissue. In a systematic review of 13 cohort studies comprising nearly 1.4 million women, the risk of preeclampsia doubled with each $5-7 \mathrm{~kg} / \mathrm{m}^{2}$ increase in prepregnancy BMI. ${ }^{21,22}$ Weight loss reduces the risk of preeclampsia and weight loss prior to pregnancy is encouraged in overweight and obese women to decrease the risk of adverse outcome.

\section{Low-dose Aspirin}

Obese women with additional risk factors for development of preeclampsia may benefit from treatment with low-dose aspirin (81 mg). In 2014, the United States Preventive Services Task Force (USPSTF) reviewed the available literature and concluded that obesity, defined as BMI more than $30 \mathrm{~kg} / \mathrm{m}^{2}$, was a moderate risk factor for preeclampsia and recommended consideration of lowdose aspirin if the patient has several moderate risk factors.

\section{Gestational Diabetes}

The prevalence of GDM is significantly higher in obese women than in general obstetrical population, and the risk increased with increasing maternal weight and BMI. ${ }^{23,24}$ The increased risk of GDM is related to an exaggerated increase in Muslim resistance in the obese state. ${ }^{25}$ In a systemic review and meta-analysis found that the overall risk for GDM in obese women was 3.76 times higher than in non-obese patients with the prevalence of GDM in increasing by $0.92 \%$ for every increase of $1 \mathrm{~kg} / \mathrm{m}^{2}$ increase in BMI. ${ }^{24}$ 


\section{Indicated and Spontaneous Preterm Birth}

The literature is conflicting regarding the association between preterm delivery and obesity. Obesity increases the risk of medically indicated preterm delivery, primarily due to obesityrelated maternal disorders, such as hypertension, preeclampsia, and diabetes. In 2010, systematic review of maternal overweight and obesity and risk of preterm birth, overweight and obese women were at increased risk of induced preterm birth compared with women of normal BMI and the risk increased with increasing weight. ${ }^{26}$ Whether obesity increases the risk of spontaneous preterm birth is less clear. Swedish study observed a relationship between severity of obesity and the risk of spontaneous extremely preterm delivery (23-27 weeks) but not for very preterm or moderately preterm. ${ }^{27}$

It has been shown that there is an independent association between PCOS and spontaneous preterm birth. The precise mechanism by which PCOS modulates the risk for spontaneous preterm birth or cervical insufficiency, independent from or as part of the obesity effects, has not been well defined, but may related to changes to relaxin levels (increased) that weaken the cervical collagen matrix.

\section{Postterm Pregnancy}

There are evidence supports an association between obesity and postpartum pregnancy (OR 1.2-1.7) beyond 41 weeks and 42 weeks gestation. ${ }^{23,28}$ The mechanism by which obesity prolongs pregnancy has not been determined. One hypothesis is that gestational age calculated from last menstrual period (LPM) overestimates true fetal age because obese women tend to be oligo-ovulatory. This hypothesis is supported by studies of early ultrasound assessment of gestational age in this population that found the expected day of delivery (EDD) by LMP was earlier than the EDD by ultrasound. ${ }^{29,30}$

\section{Multifetal Pregnancy}

It has been observed an increased incidence of dizygotic but not monozygotic, twins among obese gravidas. In an analysis of 51,783 pregnancies (561 twin) in the Collaborative Perinatal Project, the incidence of dizygotic twins in women with BMI more than 30 $\mathrm{kg} / \mathrm{m}^{2}$ and less than $25 \mathrm{~kg} / \mathrm{m}^{2}$ was $1.1 \%$ and $0.5 \%$ respectively. ${ }^{31}$ These data were derived from patients in 12 hospitals in the USA. The association of obesity with dizygotic twinning has been attributed to elevated follicle-stimulating hormone (FSH) levels in obese women.

\section{Intrapartum Complications of Obesity in Pregnancy Induction}

Obese women are at increased risk for labor induction due to their increased risk for pregnancy complications and then are at increased risk for induction failure. In one study, obese women overall were twice as likely to experience a failed induction as normal-weight women and the risk increased with increasing class of obesity. ${ }^{32}$ Induction of labor in obese women, takes longer than spontaneous labor (which is already longer), labor duration and progress inversely related to maternal weight and failure to respond to prostaglandin cervical ripening. Obese women are poorer responders to oxytocin during induction and for each additional $10 \mathrm{~kg}$ of maternal weight, $17 \%$ increase in risk of cesarean in these induction RCT obese women significantly more likely to fail oxytocin augmentation (require cesarean for dystocia despite augmentation).

\section{Cesarean Section Delivery}

Obesity is a risk factor for both elective and emergency cesarean delivery and the risk increases with increasing maternal weight and BMI. Obesity related pregnancy complications, higher infant birth weight, and increased frequency of preterm and postterm delivery account for some of the excess risk of cesarean delivery. ${ }^{33,34}$

However, obesity appears to be an independent risk factor for cesarean section. One meta-analysis demonstrated obese had a cesarean section risk that was 2.05 times higher than patients with normal weight (OR 1.86-2.27] and severe obese gravidas had a cesarean section risk that was 2.89 times higher than normal weight parents (OR 2.28-3.79). ${ }^{35}$

Cesarean in obese gravidas can be more technically challenging with increasing operative time, higher rates of postoperative wound complications, and higher rate of inflection, prolonged hospitalization, clotting disorders, and respiratory airways complications.

One-third of maternal deaths associated with obesity complications may following cesarean delivery. Observational studies have consistently reported that a trial of labor after a cesarean delivery is less likely to result in vaginal birth in obese women and had been found that increasing BMI to be inversely associated with successful trial of labor after cesarean section.

\section{Anesthesia: Complications}

Placing regional anesthesia has been shown to be more difficult in obese women, often requiring multiple attempts at needle insertion and more frequently resulting in failure of regional anesthesia placement. Intubation for general anesthesia can also be more difficult in obese patients.

\section{Timing and Route of Delivery}

Delivery by the estimated due date has been recommended to reduce the risk of stillbirth and complications from continued fetal growth. The following criteria were used to be delivered by the due date (1) prepregnancy BMI more than $40 \mathrm{~kg} / \mathrm{m}^{2}$, (2) prepregnancy BMI $35-39.9 \mathrm{~kg} / \mathrm{m}^{2}$ plus GDM or macrosomia, or (3) prepregnancy BMI $30-34.9 \mathrm{~kg} / \mathrm{m}^{2}$ plus gestational diabetes and LGA fetus. ${ }^{36}$ This protocol did not increase cesarean section rate. Fetal death does not change. Induction by the estimated date of delivery is reasonable and does not appear to increase cesarean delivery. The route of delivery should be based on standard obstetric indications. Planned cesarean delivery is not associated with less morbidity than planned vaginal delivery. ${ }^{37}$

\section{Complications Related to Macrosomia}

Obesity has been shown to be associated with prolonged or dysfunctional labor and is a well-established risk factor for fetal LGA, conferring between a 2-fold and 3-fold increased risk. Several studies have reported that increasing prepregnancy weight as a result, the obese gravida is at increased risk of delivering a LGA infant and may be related to maternal and fetal hyperinsulinemia. ${ }^{38-40} \mathrm{This}$ relationship persists even after adjusting for gestational diabetes and gestational weight gain and has also been described among obese adolescent gravidas. 
The literature is conflicting regarding the risk of shoulder dystocia among obese gravidas. Two large retrospective cohort studies found that shoulder dystocia risk is increased among obese women, an even larger population-based cohort study, including more than 400,000 pregnant women found obesity to be associated with increased rates of macrosomia but not an increased incidence of shoulder dystocia. ${ }^{41}$

Other potential intrapartum complications of macrosomia include dysfunctional labor, operative intervention (forceps or vacuum vaginal delivery, cesarean section), maternal genital tract laceration, and postpartum hemorrhage.

\section{Postpartum Complications of Obesity \\ Thromboprophylaxis}

Obesity, the postpartum state, and cesarean delivery are independent risk factors for venous thromboembolism (VTE). American College of Obstetricians and Gynecologists (ACOG) endorses universal use of pneumatic compression devices at the time of cesarean delivery and both mechanical and pharmacologic thromboprophylaxis in woman at high risk of VTE undergoing cesarean. The risk for postpartum VTE in women with class I, II, and III obesity was OR 2.5, 2.9, and 4.6, respectively, compared with women whose BMI was normal. ${ }^{42}$

\section{Childhood Obesity}

Obese parent increases the risk of obesity by 2-fold to 3-fold, and up to 15 -fold if both parents are obese. Maternal obesity is important because ultrauterine nutritional excess and development in such an environment may lead to permanent changes of fetal metabolic pathways and thereby increase the risk of childhood and adult diseases related to these pathways, such as obesity, diabetes, hypertension, and cardiovascular disease. However shared genetic or familiar lifestyle also plans a role.

\section{Congenital Anomalies}

Obese women are at increased risk for having a fetus with congenital anomalies comparing with pregnancies in women with a normal BMI. The risk appears to increase with an increasing degree of maternal obesity. Congenital anomalies including neural tube defects (NTDs), cardiac malformations, facial defects, and limb reduction anomalies (OR 1.34). ${ }^{43}$ The mechanism is not well defined but is likely related to an altered nutritional milieu during fetal development. Two meta-analyses have been recently published and document an increased risk of NTDs in fetuses of the obese gravida with pooled ORs of 1.70 and 1.87, spina bifida (OR 2.24), hydrocephaly (OR 1.68), cardiovascular anomalies (OR 1.30), septal anomalies (OR 1.20), cleft palate (OR 1.23), cleft lip and palate (OR 1.20), anorectal atresia (OR 1.48), and limp reduction anomalies (OR 1.34).

In contrast, the risk of gastroschisis was significantly reduced (OR 0.17). Systematic reviews have shown that as the severity of maternal obesity increased the risk for NTD and congenital heart defects also increased. ${ }^{44}$ Obese women do not experience the typical reduction in NTD risk associated with standard doses of folic acid. Supplementation, suggesting that folate deficiency may not be the underlying etiology of NTDs in obese women. ${ }^{45}$ It has been demonstrated that bariatric surgery does not decrease congenital anomalies.

\section{Management of Obesity: in General}

First-line of management: Lifestyle damages like modification of diet, physical activity, and daily habits.

Second-line of management: Introduction of pharmacotherapy for patients with BMI more than $25 \mathrm{~kg} / \mathrm{m}^{2}$.

Third-line of management: Bariatric surgery for treatment of extreme obesity.

\section{Prepregnancy Weight Management}

Ideally, weight loss with the goal of a normal BMI should be attempted before conception in order to plan reproduction. Obese women and the care providers should discuss about the adverse effects of obesity on fertility, the potential pregnancy complication associated with obesity and about the benefits of weight loss before attempting to conceive. ACOG recommends weight loss through a healthy diet of caloric restriction in combination with aerobic exercise.

Obese women are encouraged to undertake a weight reduction program and possibly adjunctive medical therapy or bariatric surgery if indicated before attempting to conceive, because weight loss appears to have beneficial effects on reproductive function, pregnancy outcome, and overall health. ${ }^{46}$

Bariatric surgery is not the first-line of treatment. Bariatric surgery refers to a heterogeneous group of procedures that include laparoscopic adjustable gastric bounding, vertical banded gastroplasty (restrictive procedures decrease the stomach capacity) Roux-en-Y gastric bypass, and biliopancreatic diversion/duodenal switch. (malabsorptive procedures decrease absorption of calories and nutrients by shortening functional length of small intensive). Such procedures are appropriate for women with BMI of $40 \mathrm{~kg} / \mathrm{m}^{2}$ or greater, or with BMI of $35 \mathrm{~kg} / \mathrm{m}^{2}$ or greater with comorbidities, such as diabetes, coronary artery disease, and severe sleep apnea.

\section{ConCLUSION}

- Obesity in pregnancy is best defined as prepregnancy BMI more than $30 \mathrm{~kg} / \mathrm{m}^{2}$.

- Adipose tissue is an active endocrine organ and when present in excess it can have dysregulatory effects on metabolic, vascular and inflammatory pathways in many organ systems.

- Compared with pregnant women with BMI less than $25 \mathrm{~kg} / \mathrm{m}^{2}$, pregnancies among obese women are at increased risk of early pregnancy loss, congenital anomalies, stillbirth, pregnancyassociated hypertension, preterm and postterm birth, GDM, multifetal gestation, and birth of a LGA infant. Macrosomia may result in shoulder dystocia or cesarean delivery.

- Obese pregnant women are also at increased risk for maternal disorders, such as sleep-related breathing disorders, carpal tunnel syndrome, postpartum depression, and VTE.

- Modifications to routine prenatal care have been suggested for this population:

- Screening for diabetes in early pregnancy

- Limiting gestational weight gain

- Routine ultrasound for gestational age and fetal anatomic survey

- Fetal echocardiography

- Screening for fetal aneuploidy. Obesity can affect screening test performance. Cell-free fetal DNA screening is more likely to result in test failure. 
- Low-dose aspirin to reduce the risk of preeclampsia.

- Evaluation by anesthesiologist.

- Improved pregnancy outcome with routine use of antenatal fetal surveillance with NST and BPP scoring.

- Delivery timing and indications for labor induction should not be altered solely based on maternal obesity.

- For women undergoing cesarean delivery, prophylactic antibiotics should be administered based on maternal weight.

- Pneumatic compression devices should be used to prevent postpartum VTE in all women undergoing cesarean delivery, and both mechanical and pharmacologic thromboprophylaxis should be administered to women at high risk of VTE. (UpToDate 2017 Obesity in pregnancy: complications and maternal management) Nov. 7, 2017 [online] Available from https://www. uptodate.com/contents/obesity-in-pregnancy-complicationsand-maternal-management.

\section{OrCID}

Aris Antsaklis 맘 https://orcid.org/0000-0002-3855-5496

\section{References}

1. Gallagher D, Visser M, Sepulveda D, et al. How useful is body mass index for comparison of body fatness across age, sex, and ethnic groups? Am J Epidemiol 1996;143(3):228. DOI: 10.1093/oxfordjournals. aje.a008733.

2. Mei Z, Grummer-Strawn LM, Pietrobelli A, et al. Validity of body mass index compared with other body-composition screening indexes for the assessment of body fatness in children and adolescents. Am J Clin Nutr 2002;75(6):978-985. DOI: 10.1093/ajcn/75.6.978.

3. Prospective Studies Collaboration, Lewington $S$, Sherliker $P$, et al. Body-mass index and cause-specific mortality in 900000 adults: collaborative analyses of 57 prospective studies. Lancet 2009;373(9669):1083-1096. DOI: 10.1016/S0140-6736(09)60318-4.

4. Ramsay JE, Ferrell WR, Crawford L, et al. Maternal obesity is associated with dysregulation of metabolic, vascular, and inflammatory pathways. J Clin Endocrinol Metab 2002;87(9):4231-4237. DOI: 10.1210/jc.2002-020311.

5. Catalano PM, Shankar K. Obesity and pregnancy: mechanisms of short term and long term adverse consequences for mother and child. BMJ 2017;356:j1. DOI: 10.1136/bmj.j1.

6. Tyrrell J, Richmond RC, Palmer TM, et al. Genetic evidence for causal relationships between maternal obesity-related traits and birth weight. JAMA 2016;315(11):1129-1140. DOI: 10.1001/jama.2016.1975.

7. Institute of medicine (US) and national research council (US) committee to reexamine IOM pregnancy weight guidelines. In: Rasmussen KM, Yaktine AL, ed. Weight Gain During Pregnancy: Reexamining the Guidelines. Washington, DC: National Academies Press (US); 2009.

8. Cedergren MI. Maternal morbid obesity and the risk of adverse pregnancy outcome. Obstet Gynecol 2004;103(2):219-224. DOI: 10.1097/01.AOG.0000107291.46159.00.

9. American College of Obstetricians and Gynecologists. ACOG committee opinion no. 549: obesity in pregnancy. Obstet Gynecol 2013;121(1):213-217. DOI: 10.1097/01.AOG.0000425667.10377.60.

10. Barker DJP, Osmond C, Winter PD, et al. Weight in infancy and death from ischaemic heart disease. Lancet 1989;2(8663):577-580. DOI: 10.1016/S0140-6736(89)90710-1.

11. Paneth N, Susser M. Early origin of coronary heart disease (the "Barker hypothesis"). BMJ 1995;310(6977):411-412. DOI: 10.1136/ bmj.310.6977.411.

12. Heslehurst N, Simpson H, Ells LJ, et al. The impact of maternal BMI status on pregnancy outcomes with immediate short-term obstetric resource implications: a meta-analysis. Obes Rev 2008;9(6):635-683. DOI: 10.1111/j.1467-789X.2008.00511.X.

13. Birdsall KM, Vyas S, Khazaezadeh N, et al. Maternal obesity: a review of interventions. Int J Clin Pract 2009;63(3):494-507. DOI: 10.1111/j.17421241.2008.01910.x

14. Lu GC, Rouse DJ, DuBard M, et al. The effect of the increasing prevalence of maternal obesity on perinatal morbidity. Am J Obstet Gynecol 2001;185(4):845-849. DOI: 10.1067/mob.2001.117351.

15. Stothard KJ, Tennant PWG, Bell R, et al. Maternal overweight and obesity and the risk of congenital anomalies: a systematic review and meta-analysis. JAMA 2009;301(6):636-650. DOI: 10.1001/ jama.2009.113.

16. Bellver J, Melo MAB, Bosch E, et al. Obesity and poor reproductive outcome: the potential role of the endometrium. Fertil Steril 2007;88(2):446-451. DOI: 10.1016/j.fertnstert.2006.11.162.

17. Esakoff TF, Sparks TN, Kaimal AJ, et al. Diagnosis and morbidity of placenta accrete. Ultrasound Obstet Gynecol 2011;37(3):324-327. DOI: $10.1002 /$ uog.8827.

18. Khong TY. The pathology of placenta accreta, a worldwide epidemic. J Clin Pathol 2008;61(12):1243-1246. DOI: 10.1136/jcp.2008. 055202.

19. Catalano PM, Kirwan JP, Haugel-de Mouzon S, et al. Gestational diabetes and insulin resistance: role in short- and long-term implications for mother and fetus. J Nutr 2003;133(5):1674S-1683SS. DOI: 10.1093/jn/133.5.1674S.

20. Gaillard R, Steegers EA, Hofman A, et al. Associations of maternal obesity with blood pressure and the risks of gestational hypertensive disorders. The generation R study. J Hypertens 2011;29(5):937-944. DOI: 10.1097/HJH.0b013e328345500c.

21. Silver RM, Landon MB, Rouse DJ, et al. Maternal morbidity associated with multiple repeat cesarean deliveries. Obstet Gynecol 2006;107(6):1226-1232. DOI: 10.1097/01.AOG.0000219750.79480.84.

22. Sibai BM, Ewell M, Levine RJ, et al. Risk factors associated with preeclampsia in healthy nulliparous women. The calcium for preeclampsia prevention (CPEP) study group. Am J Obstet Gynecol 1997;177(5):1003-1010. DOI: 10.1016/S0002-9378(97)70004-8.

23. Gross T, Sokol RJ, King KC. Obesity in pregnancy: risks and outcome. Obstet Gynecol 1980;56:446-450.

24. Torloni MR, Betrán AP, Horta BL, et al. Prepregnancy BMI and the risk of gestational diabetes: a systematic review of the literature with meta-analysis. Obes Rev 2009;10(2):194-203. DOI: 10.1111/j.1467789X.2008.00541.x.

25. Chu SY, Callaghan WM, Kim SY, et al. Maternal obesity and risk of gestational diabetes mellitus. Diabetes Care 2007;30(8):2070-2076. DOI: $10.2337 / \mathrm{dc} 06-2559 \mathrm{a}$.

26. Lockwood CJ, Huang SJ, Chen CP, et al. Decidual cell regulation of natural killer cell-recruiting chemokines: implications for the pathogenesis and prediction of preeclampsia. Am J Pathol 2013;183(3):841-856. DOI: 10.1016/j.ajpath.2013.05.029.

27. McDonald SD, Han Z, Mulla S, et al. Overweight and obesity in mothers and risk of preterm birth and low birth weight infants: systematic review and meta-analyses. BMJ 2010;341(jul20 1):c3428. DOI: 10.1136/ bmj.c3428.

28. Palomba S, Falbo A, Chiossi G, et al. Low-grade chronic inflammation in pregnant women with polycystic ovary syndrome: a prospective controlled clinical study. J Clin Endocrinol Metab 2014;99(8):29422951. DOI: 10.1210/jc.2014-1214.

29. Denison F, Price J, Graham C, et al. Maternal obesity, length of gestation, risk of postdates pregnancy and spontaneous onset of labour at term. BJOG 2008;115(6):720-725. DOI: 10.1111/j.14710528.2008.01694.x.

30. Simic $M$, Wåhlin IA, Marsál K, et al. Maternal obesity is a potential source of error in mid-trimester ultrasound estimation of gestational age. Ultrasound Obstet Gynecol 2010;35(1):48-53. DOI: 10.1002/ uog.7502.

31. Naeye RL. Maternal body weight and pregnancy outcome. Am J Clin Nutr 1990;52(2):273-279. DOI: 10.1093/ajcn/52.2.273. 
32. Nylander PP. The factors that influence twinning rates. Acta Genet Med Gemellol (Roma) 1981;30(3):189-202. DOI: 10.1017/ S0001566000007650.

33. O'Reilly JR, Reynolds RM. The risk of maternal obesity to the longterm health of the offspring. Clin Endocrinol (Oxf) 2013;78(1):9-16. DOI: 10.1111/cen.12055.

34. Brost BC, Goldenberg RL, Mercer BM, et al. The preterm prediction study: association of cesarean delivery with increases in maternal weight and body mass index. Am J Obstet Gynecol 1997;177(2):333337. DOI: 10.1016/S0002-9378(97)70195-9.

35. Lo TK, Yung WK, Lau WL, et al. Planned conservative management of placenta accreta-experience of a regional general hospital. J Matern Fetal Neonatal Med 2014;27(3):291-296. DOI: 10.3109/14767058.2013.818118.

36. Cohen WR, Hayes-Gill B. Influence of maternal body mass index on accuracy and reliability of external fetal monitoring techniques. Acta Obstet Gynecol Scand 2014;93(6):590-595. DOI: 10.1111/aogs.12387.

37. Schuster M, Madueke-Laveaux OS, Mackeen AD, et al. The effect of the MFM obesity protocol on cesarean delivery rates. Am J Obstet Gynecol 2016;215(4):492.e1-6. DOI: 10.1016/j.ajog.2016.05.005.

38. Yao R, Ananth CV, Park BY, et al. Obesity and the risk of stillbirth: a population-based cohort study. Am J Obstet Gynecol 2014;210(5):457. e1-9. DOI: 10.1016/j.ajog.2014.01.044.

39. Frentzen BH, Dimperio DL, Cruz AC. Maternal weight gain: effect on infant birth weight among overweight and average-weight low-income women. Am J Obstet Gynecol 1988;159(5):1114-1117. DOI: 10.1016/0002-9378(88)90424-3.

40. Calandra C, Abell DA, Beischer NA. Maternal obesity in pregnancy. Obstet Gynecol 1981;57:8-12.

41. Jauniaux E, Jurkovic D. Placenta accreta: pathogenesis of a 20th century iatrogenic uterine disease. Placenta 2012;33(4):244-251. DOI: 10.1016/j.placenta.2011.11.010.

42. Kevane B, Donnelly J, D'Alton M, et al. Risk factors for pregnancyassociated venous thromboembolism: a review. J Perinat Med 2014;42(4):417-425. DOI: 10.1515/jpm-2013-0207.

43. Molyneaux E, Poston L, Ashurst-Williams S, et al. Obesity and mental disorders during pregnancy and postpartum: a systematic review and meta-analysis. Obstet Gynecol 2014;123(4):857-867. DOI: 10.1097/ AOG.0000000000000170.

44. Rasmussen SA, Chu SY, Kim SY, et al. Maternal obesity and risk of neural tube defects: a meta-analysis. Am J Obstet Gynecol 2008;198(6):611-619. DOI: 10.1016/j.ajog.2008.04.021.

45. Cai G, Sun X, Zhang L, et al. Association between maternal body mass index and congenital heart defects in offspring: a systematic review. Am J Obstet Gynecol 2014;211(2):91-117. DOI: 10.1016/j. ajog.2014.03.028.

46. Wolfe BM, Kvach E, Eckel RH. Treatment of obesity: weight loss and bariatric surgery. Circ Res 2016;118(11):1844-1855. DOI: 10.1161/ CIRCRESAHA.116.307591. 